OPEN ACCESS

Edited by:

Sabine Wislet,

University of Liège, Belgium

Reviewed by:

Guillermo A. Vega-Lopez, Universidad Nacional de Tucumán,

Argentina

Ulf Strauss,

Charité Medical University of Berlin,

Germany

*Correspondence:

Marianna Szemes

m.szemes@bristol.ac.uk

Karim Malik

k.t.a.malik@bristol.ac.uk

Received: 19 December 2018

Accepted: 21 March 2019

Published: 16 April 2019

Citation:

Szemes M, Greenhough A and Malik K (2019) Wnt Signaling Is a Major Determinant of Neuroblastoma

Cell Lineages.

Front. Mol. Neurosci. 12:90. doi: 10.3389/fnmol.2019.00090

\section{Wnt Signaling Is a Major Determinant of Neuroblastoma Cell Lineages}

\author{
Marianna Szemes*, Alexander Greenhough and Karim Malik* \\ Cancer Epigenetics Laboratory, School of Cellular and Molecular Medicine, University of Bristol, Bristol, United Kingdom
}

The neural crest (NC), which has been referred to as the fourth germ layer, comprises a multipotent cell population which will specify diverse cells and tissues, including craniofacial cartilage and bones, melanocytes, the adrenal medulla and the peripheral nervous system. These cell fates are known to be determined by gene regulatory networks (GRNs) acting at various stages of NC development, such as induction, specification, and migration. Although transcription factor hierarchies and some of their interplay with morphogenetic signaling pathways have been characterized, the full complexity of activities required for regulated development remains uncharted. Deregulation of these pathways may contribute to tumorigenesis, as in the case of neuroblastoma, a frequently lethal embryonic cancer thought to arise from the sympathoadrenal lineage of the NC. In this "Hypothesis and Theory" article, we utilize the next generation sequencing data from neuroblastoma cells and tumors to evaluate the possible influences of Wnt signaling on NC GRNs and on neuroblastoma cell lineages. We propose that Wnt signaling is a major determinant of regulatory networks that underlie mesenchymal/neural crest cell (NCC)-like cell identities through PRRX1 and YAP/TAZ transcription factors. Furthermore, Wnt may also co-operate with Hedgehog signaling in driving proneural differentiation programmes along the adrenergic (ADRN) lineage. Elucidation of Signaling Regulatory Networks can augment and complement GRNs in characterizing cell identities, which may in turn contribute to the design of improved therapeutics tailored to primary and relapsing neuroblastoma.

Keywords: Wnt signaling, neuroblastoma, neural crest, gene regulatory networks, Hippo YAP/TAZ, Hedgehog signaling

\section{INTRODUCTION}

Neuroblastoma (NB) is a frequently lethal pediatric tumor, with $75 \%$ of NBs occurring in children under 5 years of age. About half of the tumors arise in the adrenal medulla, with the remainder originating in the paraspinal sympathetic ganglia in the abdomen or chest, or in pelvic ganglia. This distribution reflects the probable developmental origin of NB in the sympathoadrenal (SA) lineage of the neural crest (NC). Clinically, NB encompasses low-risk disease which responds well to treatment and may even spontaneously regress, and high-risk disease, representing about $40 \%$ of total cases, which frequently relapse and have less than $50 \%$ survival.

\footnotetext{
Abbreviations: ADRN, adrenergic; EMT, epithelial-mesenchymal transition; ERK, extracellular signal-regulated kinase; GRN, gene regulatory network; KEGG, Kyoto encyclopedia of genes and genomes; LEF, lymphoid enhancer binding factor; MEK, MAPK/ERK kinase; MES, mesenchymal; MNA, MYCN-amplified; NB, neuroblastoma; NC, neural crest; NCC, neural crest cell; Rspo, R-spondin; SA, sympathoadrenal; TCF, T-cell-factor.
} 
This clinical heterogeneity is thought to reflect both the complex molecular etiology as well as the cellular heterogeneity of tumors (Brodeur, 2003; Maris et al., 2007). Whilst it has long been known that the proto-oncogene $M Y C N$ is crucial for NC cell fate (Wakamatsu et al., 1997) and influencing differentiation states in NB (Westermark et al., 2011), a deeper understanding of developmental factors remains necessary to determine the origins of $\mathrm{NB}$ in order to inform improved prognosis and therapies.

In this theoretical analysis, we will consider the potential regulatory influences and interactions of the canonical Wnt signaling pathway in determining phenotypes in the NC and neuroblastoma. Although this signaling pathway is known to be critical in regulating stemness, cell fate, differentiation and proliferation (Nusse and Clevers, 2017), much remains unclear about its role in neuroblastoma. Based on our recent identification of genes regulated by the Wnt ligand Wnt3a and Wnt agonist R-spondin 2 in an NB cell-line (Szemes et al., 2018), we assess transcriptional and signaling pathways, which require further investigation in the contexts of $\mathrm{NC}$ development and neuroblastoma.

\section{WNT SIGNALING PATHWAYS AND COMPONENTS}

In broad terms, Wnt signaling includes "canonical" and "noncanonical" pathways. The former is also referred to as $\mathrm{Wnt} / \beta$ catenin signaling, as it is dependent on cytoplasmic-nuclear translocation of $\beta$-catenin, and its subsequent transcriptional cofactor activity with T-cell factor/lymphoid enhancer factor (TCF/LEF) transcription factors (Clevers and Nusse, 2012; Nusse and Clevers, 2017). Non-canonical, or alternative Wnt signaling pathways include the Planar cell polarity (PCP), the $\mathrm{Wnt} / \mathrm{Ca}^{2+}$ pathways, $\beta$-catenin-independent pathways acting via Rho-associated kinase (ROCK) and G-protein dependent calcium release (Komiya and Habas, 2008). More recently, another alternative Wnt pathway has been demonstrated, where the downstream effectors are the Hippo signaling pathway transcriptional co-factors, Yes-associated protein (YAP) and Transcriptional C-activator with PDZ-Binding motif (TAZ, encoded by WWTR1, Park et al., 2015; Figure 1).

There is a complex and extensive interplay between these pathways, reflected in the $19 \mathrm{Wnt}$ ligands and 10 Frizzled (FZD)

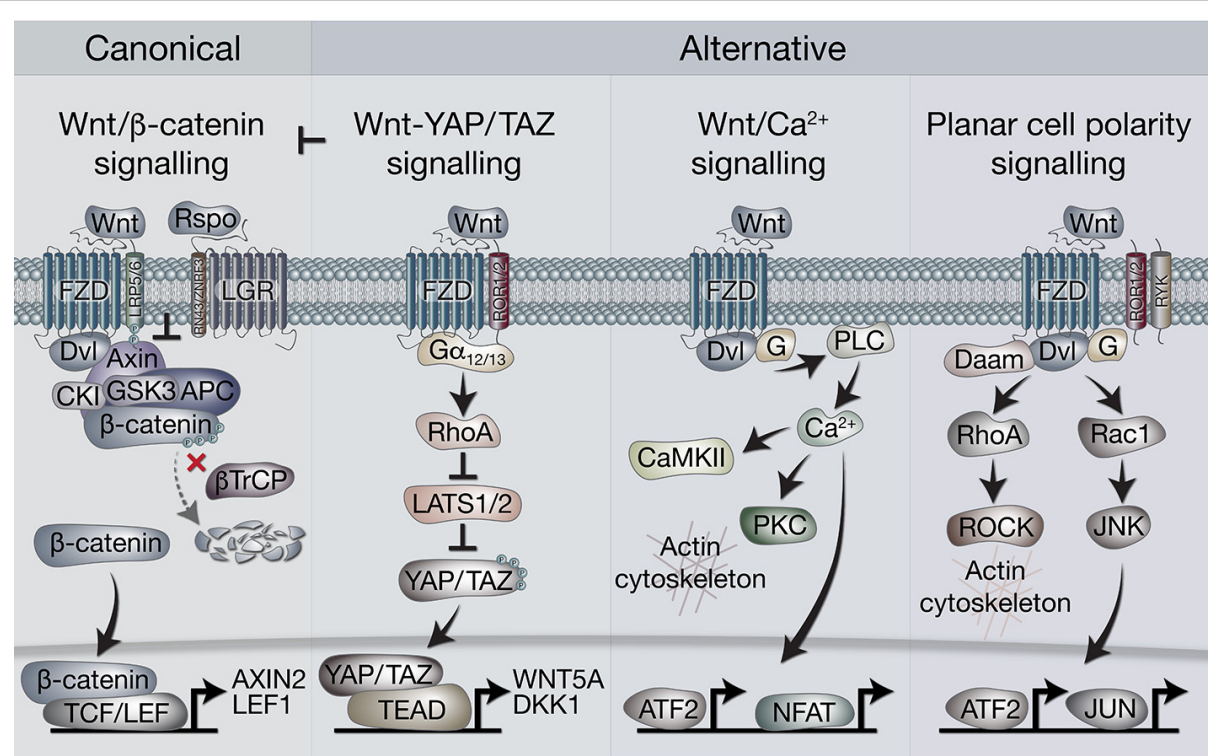

FIGURE 1 | Overview of canonical and alternative Wnt signaling pathways. Canonical Wnt signaling is defined by the activation of $\beta$-catenin-dependent transcription [via T-cell factor/lymphoid enhancer factor (TCF/LEF)] downstream of Wnt receptors. In the absence of Wnt ligands, $\beta$-catenin is held in the destruction complex of proteins that includes Axin, APC, Ser/Thr kinases CK1 and GSK3, and E3-ubiquitin ligase $\beta$-TrCP. Sequential phosphorylation (by CK1 and GSK3) and ubiquitination (by $\beta$-TrCP) of $\beta$-catenin promotes its proteosomal degradation. Binding of Wnt ligands (classically Wnt3a) to frizzled (FZD) and LRP5/6 receptors leads to recruitment of the destruction complex to the membrane via Dvl and Axin, which blocks $\beta$-catenin ubiquitination by $\beta$-TrCP. The destruction complex then becomes saturated with $\beta$-catenin, allowing newly synthesized $\beta$-catenin to accumulate in the cytoplasm and translocate to the nucleus where it associates with TCF/LEF transcription factors to regulate gene expression. Rspo (R-spondin) proteins can enhance Wnt signaling by binding LGR4/5/6 receptors to antagonize the RNF43/ZNRF3 transmembrane E3 ligases that remove Wnt receptors from the cell surface. The alternative Wnt-YAP/TAZ pathway (as defined by Park et al., 2015). Wnt5a/b and Wnt3a ligands induce the activation of YAP/TAZ via FZD and ROR1/2 co-receptors, independently of LRP5/6 and $\beta$-catenin. Activation of FZD-ROR1/2 couples to Ga12/13 G-protein subunits, leading to activation of RhoA and subsequent inhibition of LATS1/2 kinases (major YAP/TAZ Ser/Thr kinases). Inhibition of LATS1/2 leads to YAP/TAZ dephosphorylation, stabilization and translocation to the nucleus where their interaction with TEAD transcription factors promotes gene regulation. Wnt-YAP/TAZ target genes such as DKK1 lead to the inhibition of canonical Wnt/ $\beta$-catenin signaling. Wnt/Ca ${ }^{2+}$ signaling. Activation of FZD stimulates the activity of phospholipase C (PLC) via Dvl and G proteins, leading to increases in intracellular $\mathrm{Ca}^{2+}$ levels. Ca ${ }^{2+}$ activates calmodulin-dependent protein kinase II (CaMKII), protein kinase C (PKC) and the transcription factors NFAT and ATF2. Activation of PKC by Ca ${ }^{2+}$ can cause actin cytoskeleton rearrangements via Cdc42 activity. Wnt/PCP (planar cell polarity) signaling. Wnt signaling via FZD with co-receptors ROR1/2 or RYK may activate G proteins and Dvl to stimulate RhoA (via Dvl-Daam) and ROCK activity, leading to rearrangement of the actin cytoskeleton. Activation of G proteins can also stimulate Rac1, leading to JNK-mediated phosphorylation of C-JUN and transcriptional activity via AP-1. 
receptors. Wnt ligands are secreted, lipid-modified glycoproteins conserved in all metazoan animals, able to act as morphogens over short distances (MacDonald et al., 2009). Although some preferential usage of certain Wnt ligands by different pathways is observed, such as Wnt3a for canonical, and Wnt5a for non-canonical signaling, strict restriction of ligand usage by pathways, or a "Wnt code," have not emerged. Rather, numerous other effectors and regulatory molecules add to the complexity of the pathways (van Amerongen, 2012). In general, however, non-canonical Wnt signaling is thought to antagonize the canonical Wnt/ $\beta$-catenin pathway (Veeman et al., 2003).

In the canonical Wnt/ $\beta$-catenin pathway, Wnt ligands are bound with high affinity by Frizzled proteins, leading to the formation of heterodimeric membrane core receptor complexes containing LRP5/6. Binding of Wnt ligands leads to LRP phosphorylation and recruitment of the scaffold protein Axin to LRP. Axin is a key component of the "destruction complex," which also includes APC, the Ser/Thr kinases GSK-3 and CK1, protein phosphatase 2A (PP2A), and the E3-ubiquitin ligase $\beta$-TrCP. This complex regulates cytoplasmic $\beta$-catenin turnover through phosphorylation and proteosomal turnover. The LRP-Axin interaction leads to inactivation of the destruction complex, permitting $\beta$-catenin stabilization and nuclear availability. The TCF/LEF family of transcription factors are then able to utilize $\beta$-catenin as a transcriptional co-activator and instigate target gene expression (Clevers and Nusse, 2012). Further amplification of signaling can be achieved through the participation of another set of receptors, the leucine-rich repeat-containing G-protein coupled receptors (LGR4/5/6) and their ligands, the R-spondins (Rspos; de Lau et al., 2011). LGR-Rspo complexes at the cell membrane decrease the endocytic turnover of Frizzled-LRP5/6 by neutralizing the ubiquitin ligases RNF43 and ZNRF3 (Hao et al., 2012).

\section{DIFFERENTIATION OF THE NEURAL CREST AND WNT SIGNALING}

The tissue of origin for neuroblastoma is the NC, a band of cells that forms transiently between the neural tube and the non-neural ectoderm during early vertebrate development. Many signaling pathways act together during gastrulation to promote NC induction and specification, especially bone morphogenetic protein (BMP; Steventon et al., 2009), fibroblast growth factor (FGF; Stuhlmiller and García-Castro, 2012) and Wnt/ $\beta$-catenin signaling (García-Castro et al., 2002; Leung et al., 2016). Together, these signaling pathways orchestrate numerous transcription factors, including MSX1/2 (Tribulo et al., 2003; Ramos and Robert, 2005) and ETS1 (Barembaum and Bronner, 2013). NC specification finishes with an elevation of the neural folds during neurulation, and thereafter, following neural tube closure, premigratory NC cells delaminate and undergo epithelial-mesenchymal transition (EMT; Nieto et al., 2016). Multipotent neural crest cells (NCCs) with differential abilities to form derivative cell types are arranged along the length of the vertebrate embryo; these can be subdivided into the cranial, vagal, trunk and sacral $\mathrm{NC}$, and will give rise to diverse cell and tissue types, including facial cartilage and bone, dental mesenchymal cells (Sharpe, 2016), melanocytes, smooth muscle cells, Schwann cells and enteric neurons (Bronner and LeDouarin, 2012; Martik and Bronner, 2017). The trunk NCCs give rise to the sympathetic nervous system and the adrenal medulla and are therefore the source of presumptive progenitors of neuroblastoma cells. Interestingly, a recent report by EspinosaMedina et al. (2016) demonstrated that sacral NC can also give rise to sympathetic neurons.

As well as being crucial for NC induction, canonical Wnt signaling has also been shown to be vital in other $\mathrm{NC}$ stages including a role in delamination in co-operation with BMP; here disruption of $\beta$-catenin and TCF/LEF inhibits cell cycle progression and NC delamination and transcription of BMP-regulated genes, including MSX1 (Burstyn-Cohen et al., 2004). Furthermore, ablating $\beta$-catenin specifically in NC stem cells in vivo revealed that, despite some NC-derived structures developing normally, mutant animals lack melanocytes and dorsal root ganglia. $\beta$-catenin mutant NCCs appear to emigrate normally but fail to undertake sensory neurogenesis, thereby suggesting a role of $\beta$-catenin in premigratory/early migratory NCCs (Hari et al., 2002). Non-canonical Wnt signaling is also involved in NCC migration (Mayor and Theveneau, 2014). Thus, Wnt signaling pathways are involved at multiple stages of NC development and can also selectively regulate NC lineages.

The aforementioned signaling pathways orchestrate cell behavior via gene regulatory networks (GRNs) that govern NC development. Transcriptional analysis and molecular loss and gain-of-function studies facilitate a GRN model of the NC, which, encompassing transcriptional regulators and signaling molecules, can explain the formation and maintenance of NC lineages (Martik and Bronner, 2017). Establishment of the neural plate border, specification, migration and differentiation, have their specific GRNs, and these networks can interact, overlap and influence each other. Gradients and balances of Wnt, BMP, FGF and Notch signaling determine NC induction and activate transcription factors (e.g., MSX1, MYCN, $D L X 5 / 6)$, in the neural plate border module, determining the boundaries of NC, neural and non-neural ectoderm. These, in turn, upregulate NC specifier genes, which initiate the EMT programme (including SNAI1/2, ETS1, TWIST1/2) to prepare for delamination of NCCs as well as maintain an undifferentiated state (premigratory module). Following EMT, the NCCs acquire a migratory programme enabling long-distance migration guided by environmental signals and maintained by a TF network (including ZEB2, LMO4, SNAI1/2, TWIST1/2, ETS1). The sympathoadrenergic lineage follows a ventral migratory pattern and aggregate at the dorsal aorta and go on to form the primary sympathetic ganglia and colonize the adrenal medulla. $\mathrm{BMPs}$ released by the wall of the dorsal aorta induce SA speciation, activating ASCL1 and $P H O X 2 B$, which switches on other SA-lineage specific transcription factors, for example, ASCL1 upregulates $P H O X 2 A$, which in turn upregulates $T H$ and $\mathrm{DBH}$, enzymes characteristic of the SA lineage.

MYCN, the key developmental transcription factor deregulated in neuroblastoma, has been shown to be a Wnt target gene in chicken limb mesenchyme (ten Berge et al., 2008). $M Y C N$ is expressed at high levels in chick NC progenitors in 
early NC development but surprisingly is not apparent in highly proliferative migrating NCCs (Khudyakov and Bronner-Fraser, 2009). This finding was confirmed and extended to show the absence of MYCN expression in the condensed dorsal root and sympathetic ganglia. Elevating MYCN expression in the neural plate border was shown to lead to a change in NC identity, toward a more central nervous system fate (Kerosuo et al., 2018). Transient over-expression of ectopic MYCN in early migrating cells, however, suggested that $\mathrm{MYCN}$ can regulate NC fate by regulating ventral migration and neuronal differentiation (Wakamatsu et al., 1997). Evidence from the developing NC in lamprey suggests that ZIC, MSX and TFAP2A/AP2 transcription factors may positively regulate $M Y C N$ in the NC (Nikitina et al., 2008), but signaling pathways upstream of $M Y C N$ in the NC are unclear.

\section{NEUROBLASTOMA AND WNT SIGNALING}

Neuroblastomas display relatively few gene mutations, thus limiting the options for targeted therapeutics, and relapsing disease is common for high-risk NB (Matthay et al., 2016). Another increasingly important consideration is the cellular heterogeneity of NBs. Although this was first observed decades ago in NB cell lines being able to transdifferentiate (Ciccarone et al., 1989), the dependence of lineage identity on transcriptional circuitries are only recently becoming evident. One study recently established that primary NBs and NB cell lines can contain two major cellular components, including migrating, NCC-like mesenchymal (MES), and more committed adrenergic (ADRN) cells. These two cell types were defined by superenhancer-associated gene expression patterns, characteristic of NC lineage differentiation stages and could transdifferentiate in vitro and in vivo. The ADRN lineage was defined by transcription factors such as GATA2/3, PHOX2A/2B and DLK1, whereas the MES lineage included the classical EMT transcription factor SNAI2, together with YAP1, TAZ (encoded by WWTR1) and PRRX1. The ADRN cells were more tumorigenic in nude mice than MES cells, although MES cells were more chemoresistant and were enriched in relapsed tumors (van Groningen et al., 2017). Concomitantly, another group also established transcriptional circuitries underlying the heterogenous differentiation states of NB cells and termed their three subtypes as sympathetic noradrenergic, NCC-like and a mixed type (Boeva et al., 2017). The noradrenergic identity required a transcription factor module containing $\mathrm{PHOX} 2 \mathrm{~B}$, HAND2, and GATA3 and therefore corresponds to the ADRN subtype defined by van Groningen et al. (2017), whereas the NCC-like overlaps with the MES subtype, given they both require PRRX1. Importantly, ectopic overexpression of PRRX1 was shown to be able to alter lineage identity from ADRN to MES cells (van Groningen et al., 2017).

The prototypic route for disrupted differentiation of NCCs leading to neuroblastoma depends on MYCN amplification (Brodeur et al., 1984), which in turn leads to overexpressed MYCN protein directly repressing genes required for sympathetic nervous system terminal differentiation (Westermark et al., 2011; Gherardi et al., 2013). Given the many examples of cancers dependent on oncogenic Wnt/ $\beta$ catenin signaling (Clevers and Nusse, 2012), such as colorectal cancers which have activating mutations in $\beta$-catenin or loss of function APC mutations (Fodde and Tomlinson, 2010), and that MYCN is a target of Wnt signaling (ten Berge et al., 2008 ), it was reasonable to propose that $\mathrm{Wnt} / \beta$-catenin signaling would represent an oncogenic pathway in NB. This was further supported by our demonstration that high levels of LGR5 were apparent in undifferentiated NBs and NB cell lines (Vieira et al., 2015) and LGR5 expression correlated with poor prognosis. However, although we showed that LGR5 was able to function as a Rspo 2 receptor and amplify Wnt3a-induced $\beta$-catenin/TCF transcriptional activity, marked increases in proliferation were not observed, and the pro-survival functions of LGR5 were shown to be attributable to LGR5 positively modulating MEK/ERK signaling rather than Wnt/ $\beta$-catenin signaling (Vieira et al., 2015). This dual regulatory capacity of LGRs on MEK/ERK signaling and Wnt was subsequently also demonstrated in skin carcinogenesis (Xu et al., 2016), but the underlying mechanisms remain unclear.

Although we found that Wnt3a/Rspo 2 treatment of NB lines lead to the induction of some established canonical Wnt target genes, such as LEF1 and AXIN2, MYCN transcripts were not induced. In fact, we found that MYCN and MYC protein levels were actually reduced after $\mathrm{Wnt} 3 \mathrm{a} / \mathrm{Rspo} 2$ treatment, in contrast to previous reports suggesting induction of MYC in non-MYCN amplified (non-MNA) NBs as a result of $\mathrm{Wnt} / \beta$ catenin signaling (Liu et al., 2008). Furthermore, our study did not align simply with reports showing that individual Wnt pathway components were associated with chemoresistance (FZD1; Flahaut et al., 2009), tumorigenic stem-like cells in human and mouse neuroblastoma (FZD6; Cantilena et al., 2011), and increased NB proliferation dependent on FZD2 (Zins et al., 2016). Using Wnt chemical agonists and inhibitors another study suggested that Wnt signaling hyperactivation promotes apoptosis of $\mathrm{NB}$ cells and that Wnt inhibition decreased proliferation and increased NB differentiation (Duffy et al., 2016). Oncogenicity of $\mathrm{Wnt} / \beta$-catenin signaling, via deregulation of MYCN, was also inferred in a report demonstrating the therapeutic benefit of glypican-2 immunotherapy for NB ( $\mathrm{Li}$ et al., 2017).

Given the lack of consensus on the role of Wnt signaling in $\mathrm{NB}$, we, therefore, sought to detail the phenotypic and transcriptomic effects of Wnt signaling on NB cells by treating three cell lines with Wnt3a and Rspo2. We determined early (6 h) Wnt-induced transcriptomic changes by RNA sequencing in SK-N-BE(2)-C, an MYCN-amplified (MNA) NB cell line (Szemes et al., 2018). Using a statistical cut-off of $p=<0.005$, we identified 90 genes that showed substantial and significant changes. In parallel, longer treatments were assessed for changes in phenotype and established that Wnt3a/Rspo 2 treatment could induce proliferation in the SK-N-AS cell line, but in other lines, especially SK-N-BE(2)-C and SH-SY5Y cell lines, increased proliferation was not observed. Instead, we found an EMT-like transition, evidenced by increased cell migration and induction of SNAI1/2 and TWIST1/2, and neural differentiation accompanied by increased expression of marker genes such as 
NGFR, NTRK1, NEFM and NEFL/NF68. Meta-analysis of the expression of our $90 \mathrm{Wnt}$ target genes in primary tumor gene expression databases revealed: (i) four gene subsets within the 90 genes, and (ii) dramatically different prognostic outcomes associated with each of the four gene subsets (Figure 2). By converting the expression of each of the four gene subsets into a numerical model, or metagene, we were further able to show that, for example, expression of Wnt metagene 1 (WMG-1) strongly positively correlated with Hippo-YAP/TAZ signaling signatures $(R=>0.76)$ in $\mathrm{NB}$, and that these genes were more highly expressed in intermediate risk $\mathrm{NB}$, rather than low- or high-risk NB. WMG-3 was overexpressed in the high-risk NB cluster,
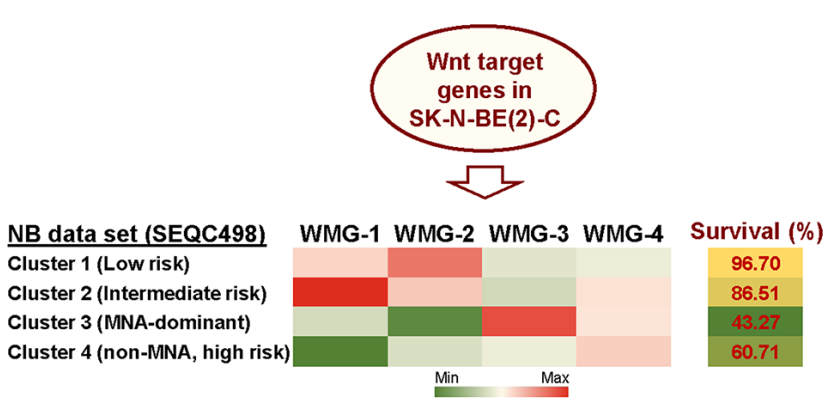

FIGURE 2 | Summary of Wnt target genes previously identified in SK-N-BE(2)-C cell line (Szemes et al., 2018). K-means clustering of these target genes in the SEQC neuroblastoma (NB) dataset (GSE62564) enabled identification of four co-expressed Wnt target gene subsets. The combined expression of each gene subset was converted into a single value, represented as a metagene. The Wnt metagene groups (WMG-1, 2, 3, 4) displayed differential expression in four prognostic clusters in the primary NB tumor data set. Expression levels of the WMGs in the NB clusters are indicated by the heatmap, and survival for each NB patient cluster is shown in the last column. Bioinformatic analyses were performed by using tools implemented in R2 (http://r2.amc.nl, http://r2platform.com). dominated by MNA tumors. Conversely, WMG-2 showed a strong positive correlation to Hedgehog signaling $(R=>0.84)$ associated with differentiation (Souzaki et al., 2010) and a negative correlation with MYC signatures $(R=-0.76)$. WMG-2 genes were, concordantly, strongly repressed in high-risk MNA NB (Szemes et al., 2018).

Collectively, studies from our laboratory and from others allude to an underappreciated role for Wnt signaling in NC and NB biology. In particular, the induction of EMT-like changes and the correlation of a subset of our Wnt target genes (WMG1) with Hippo-YAP/TAZ pathway gene signatures, seemingly characteristic of the MES lineage (van Groningen et al., 2017), suggest that Wnt signaling may be part of the regulatory network determining NB cell identity. In the following sections, we will evaluate this concept through analysis of Wnt signaling on transcriptional and signaling pathways involved in NC and NB.

\section{IS WNT SIGNALING A MAJOR DETERMINANT OF NC AND NB CELL FATE?}

Numerous studies have examined the spatiotemporal expression of transcription factors and signaling pathway components in the NC. Integration of these efforts has facilitated the establishment of a GRN model, underlying NC development (Martik and Bronner, 2017). As our RNA sequencing of the NB cell line SK$\mathrm{N}-\mathrm{BE}(2)-\mathrm{C}$ treated with $\mathrm{Wnt} 3 \mathrm{a} / \mathrm{Rspo} 2$ provides a unique gene set representing, at least in part, Wnt targets in the NC, we assessed which GRN genes encoding transcription factors or signaling molecules from different stages of NC development might be affected by Wnt3a/Rspo2 treatment (Table 1). As expected from our previous work, premigratory and migratory modules, including several EMT transcription factors (TWIST1, ZEB2, ETS1), are affected. Interestingly, given that the presumptive

TABLE 1 | Wnt3a/RSPO-2 induction in SK-N-BE(2)-C neuroblastoma cells (ERP023744; Szemes et al., 2018) can modulate gene regulatory network (GRN) modules of the developing neural crest (as identified in Martik and Bronner, 2017).

\begin{tabular}{|c|c|c|c|c|c|}
\hline Neural crest GRNs & Genes & Wnt-induced fold change & Neural crest GRNs & Genes & Wnt-induced fold change \\
\hline & BMP4 & 14.22 & \multirow{3}{*}{ Pre-migratory NC } & sox6 & 2.25 \\
\hline & WNT11 & 4.15 & & TWIST1 & 1.81 \\
\hline & WNT5B & 3.14 & & MYC & 1.32 \\
\hline & WNT4 & 3.06 & \multirow[t]{8}{*}{ Migratory NC } & WNT11 & 4.15 \\
\hline & WNT2B & 2.84 & & WNT5B & 3.14 \\
\hline & SMAD6 & 1.40 & & ETS1 & 2.56 \\
\hline & FGF18 & 1.36 & & sox6 & 2.25 \\
\hline & FGF7 & 0.71 & & TCF7 & 1.85 \\
\hline & FGF19 & 0.53 & & TWIST1 & 1.81 \\
\hline \multirow[t]{3}{*}{ Neural plate border } & MSX1 & 14.66 & & ZEB2 & 1.38 \\
\hline & $D L X 5$ & 3.54 & & LMO4 & 1.35 \\
\hline & & & Enteric neurons & ASCL1 1 & 0.58 \\
\hline
\end{tabular}

Regulatory genes with a minimum change of \pm 1.5 -fold are shown and are grouped according to the GRN modules, highlighted in different colors. 
cell of origin for NBs is along the sympathoadrenal lineage, there is also strong induction for neural plate border specifiers MSX1, DLX5 and DLX6. This suggests that either SK-N-BE(2)$C$ cells have retained early transcriptional plasticity, or more intriguingly, that NBs may arise prior to sympathoadrenal differentiation. This notion is supported by developmental experiments showing ectopic MYCN expression in the neural plate border resulting in a shift towards a CNS-like state (Kerosuo et al., 2018) and the induction of NB in MYCN-overexpressing NCCs (Olsen et al., 2017).

We next assessed the relationships between the Wnt pathway and NCCs and ARDN and MES identities using publicly available gene expression datasets. As shown in Figure 3A, transcription factors in the Wnt pathway are significantly higher in NCCs and MES cell lines compared with ADRN cell lines.

In order to explore if Wnt may regulate NB lineage identity, we studied the relationship between individual Wnt pathway members and MES and ADRN lineage identity signatures in a primary NB dataset (SEQC498; Su et al., 2014). MES and ADRN metagenes were constructed on the basis of the study of van Groningen et al. (2017). We then plotted the correlation of expression (correlation co-efficient, R) of each Wnt pathway member gene in relation to the expression of MES and ADRN signatures. In addition to the components of the Wnt pathway described in Kyoto encyclopedia of genes and genomes (KEGG), we also included LGR receptors and their ligands, R-spondins. Wnt components showed a strong correlation to the expression of MES signature along a gradient, with $R$ values ranging from 0.81 to -0.381 (Figure 3B, top panel). The association to the ADRN signature was also pronounced $(R:-0.54$ to 0.48 ), but interestingly displaying an inverse pattern to that of MES. The functional significance of this pattern could be underpinned, for example, by FZD3, which has been shown to be required for maintenance of dividing sympathetic neuroblasts and correlates with ADRN identity (Armstrong et al., 2011). In contrast, FZD1 and FZD6, previously reported to be associated with chemoresistance and tumorigenic stem-cells, respectively (Flahaut et al., 2009; Cantilena et al., 2011) correlate highly with MES identity (Figure 3B). Although the selectivity of Wnt-Frizzled interactions is poorly understood (Grainger and Willert, 2018), our analysis highlights the possibility that Wnt responses and opposing functional outcomes in NC and NB may be regulated by Wnt receptor configurations.

We previously demonstrated that Wnt can drive the expression of diverse sets of genes in NB cells that display distinct co-expression patterns in primary tumors, which we represented with Wnt metagenes. We reasoned that differential regulation of Wnt metagenes in NB tumors could be due to the configuration of the Wnt pathway, i.e., the differential expression of genes encoding for the pathway components. We, therefore, assessed the correlation of expression between Wnt pathway members and our Wnt metagenes, WMG-1, 2, 3 and 4 (Figure 3B), which revealed a striking similarity of WMG-1 (yellow) with MES identity, and an anticorrelation with ADRN identity. This was also partially true for WMG-2 (green), but to a much lesser extent, whereas WMG-3 (red), which we had previously associated with high-risk and MYC signatures, was generally

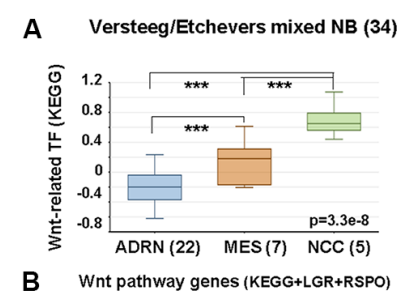

B
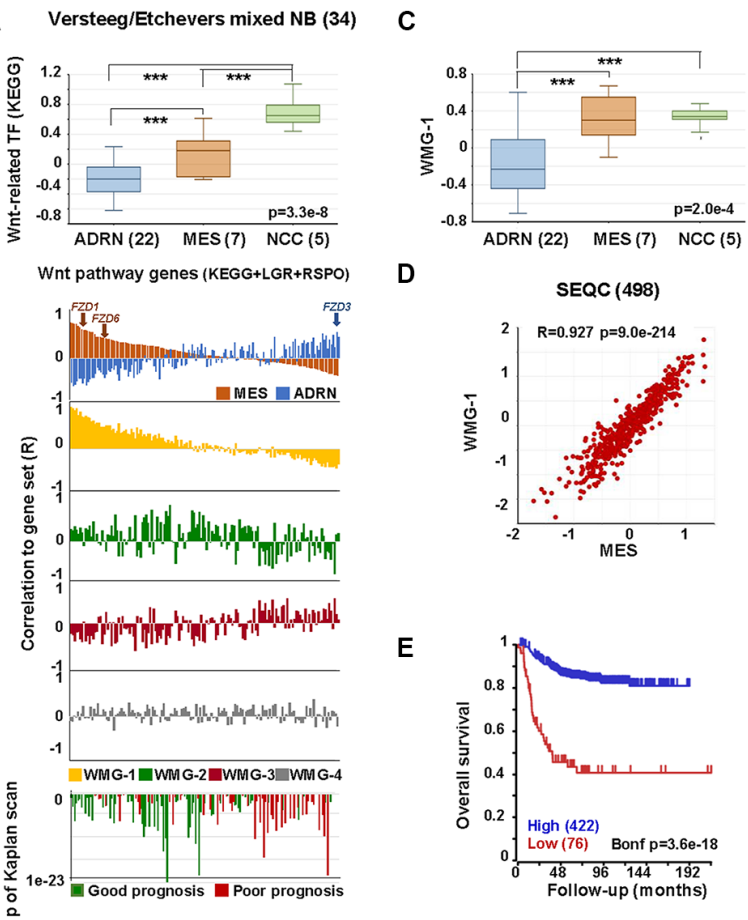

E

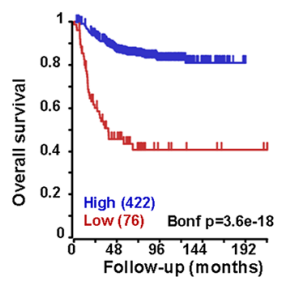

FIGURE 3 | Wht pathway associations with MES phenotype in neural crest (NC) cells and NB. (A) Expression of Wnt-related transcription factors [Kyoto encyclopedia of genes and genomes (KEGG)] in adrenergic (ADRN), mesenchymal (MES), and neural crest cell lines (NCC; GSE14340 and GSE28019, van Groningen et al., 2017). Statistically significant difference between the groups was tested by using analysis of variance (ANOVA; $p$ values shown) and Tukey HSD post hoc tests were applied to evaluate pairwise differences $(* * * 0<0.005)$. (B) Correlation of the expression of Wnt pathway members (KEGG, LGRs and RSPOs; $n=147$ ) with various gene sets and prognosis in the SEQC data set (GSE62564), sorted according to the coefficient of correlation to MES signature. The $x$-axis represents Wht pathway genes, while the $y$-axis indicates the correlation coefficient $(R)$ between the expression of the Wnt pathway gene and the indicated gene set. The upper panel shows the inverse association of MES (orange) and ADRN (blue) signatures to Wnt genes, while the lower panels show the correlations of the individual Wnt metagenes to the same pathway genes. Note the very similar pattern of correlation of WMG-1 (yellow) and MES signature to Wnt pathway genes and the inverse profile of WMG-2 (green) and WMG-3 (red) correlations. WMG-4 correlations are shown in gray. The bottom panel shows the correlation of the same genes with prognosis, with the $y$-axis representing Bonferroni corrected $p$-values of Kaplan scan analyses using event-free survival. Green bars indicate the correlation of high expression to good prognosis, while the red ones to poor prognosis. (C) WMG-1 expression in adrenergic, mesenchymal and neural crest cells. (D) Correlation between MES signature and WMG-1 in SEQC (GSE62564). Each point represents a tumor. (E) High expression of MES signature genes is correlated to good prognosis in the same data set.

low in MES and high in ADRN. WMG-2 and 3 had inverse correlation profiles, consistent with their contrasting expression patterns in NB tumors. WMG-4 (gray) correlation patterns were not distinct.

Wnt pathway members also correlated with prognosis (Figure 3B bottom). High expression of genes with moderate correlation to MES or ADRN signature and high correlation to WMG-2 were the strongest indicators of good prognosis. 
Conversely, Wnt pathway genes correlating with ADRN signature were usually indicators of poor prognosis.

Focussing on WMG-1, we observed it was also significantly higher in NCC and MES cells lines compared to ADRN cells (Figure 3C), and in the SEQC dataset WMG-1 and the MES signature showed a remarkable correlation $(R=0.927$, $p=9.0 \times 10^{-214}$; Figure 3D). This was also apparent in a second
NB dataset containing 649 tumors (Supplementary Figure S1A). We had previously shown that WMG-1 expression in NB gene expression datasets correlated with intermediate risk NB, and in accordance with that, we found that the high expression of the MES signature metagene correlated with a better prognosis in the SEQC (Figure 3E) and also in the Kocak dataset of 649 tumors (Kocak et al., 2013; Supplementary Figure S1B). As both our

\section{A}
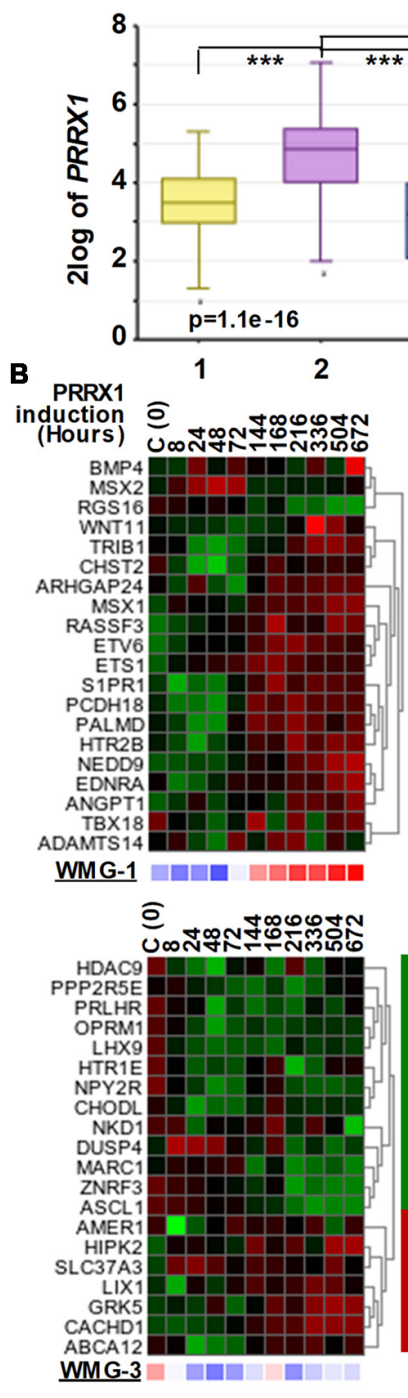

Min
NB clusters in SEQC (498)
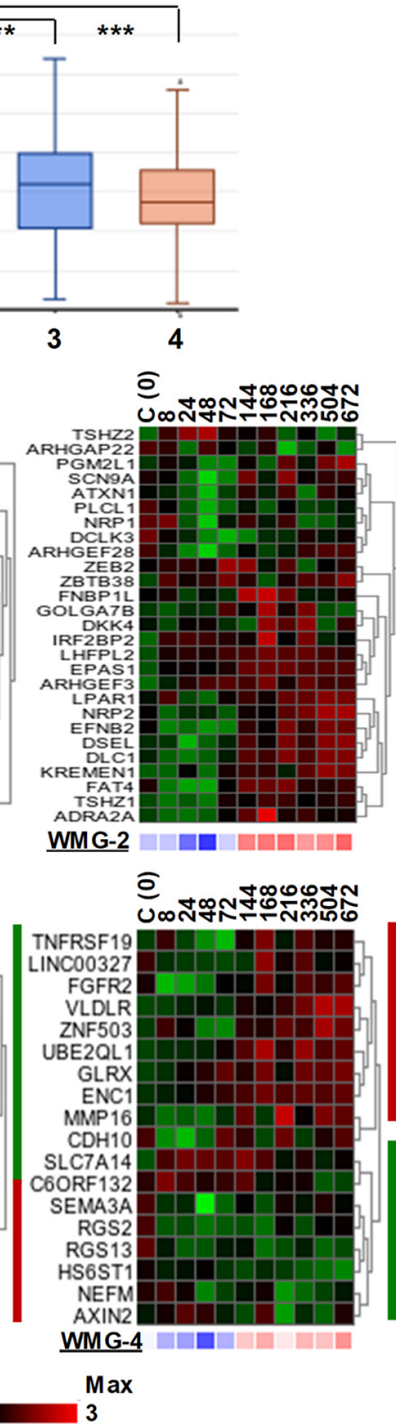

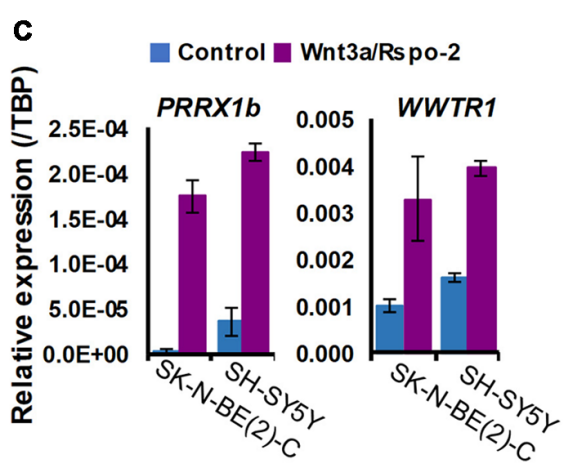

D

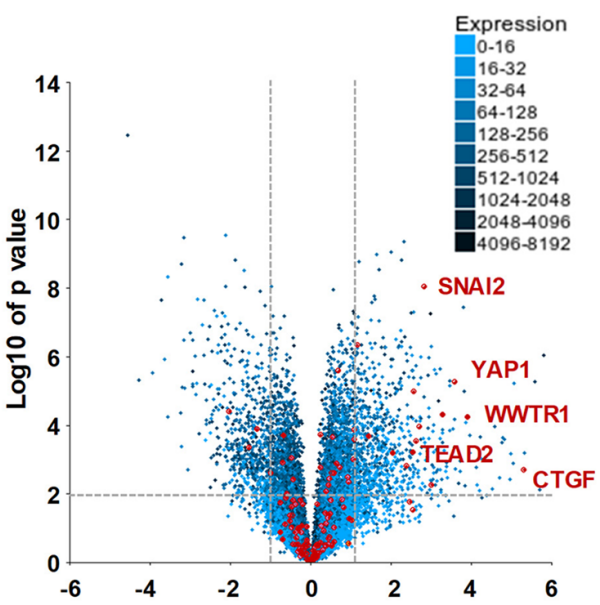

Log2 fold change of gene expression in SK-N-BE(2)-C after PRRX1 induction/control

FIGURE 4 | Associations between neuroblastoma Wnt target genes, PRRX1, and Hippo-YAP/TAZ signaling. (A) PRRX1 expression is significantly higher in the intermediate risk NB Wnt cluster, which strongly associates with high WMG-1 expression (see Figure 1; GSE62564). Statistically significant difference between the groups was tested by using ANOVA ( $p$ values shown) and Tukey HSD post hoc tests were applied to evaluate pairwise differences $(* * * p<0.005)$. (B) Heatmaps showing the regulation of individual genes within the Wnt metagenes by PRRX1 (GSE908040, derived from inducible PRRX1 expression in SK-N-BE(2)-C cells). Note the induction of WMGs 1, 2, and 4 at later timepoints, and downregulation of most WMG-3 genes. (C) Wnt3a/Rspo-2 treatment of NB cell lines for $96 \mathrm{~h}$ leads to the activation of MES-related transcription factors, PRRX1, and WWTR1. (D) Volcano plot showing gene expression changes in SK-N-BE(2)-C cells with induced PRRX1 expression. Red dots indicate members of the Hippo signaling pathway (KEGG). The highlighted Hippo pathway transcription factors and the Hippo target gene CTGF were all strongly upregulated. Dashed lines separate genes regulated by minimum \pm 2 -fold with $p<0.01$ significance. Cell culturing and gene expression analysis were performed as previously (Szemes et al., 2018). PCR primers are provided in Supplementary Table S1. 
WMG-1 (Szemes et al., 2018) and MES (van Groningen et al., 2017) signatures are associated with Hippo-YAP/TAZ, it may be inferred that Hippo signaling, which has been implicated in NB tumorigenesis (Wang et al., 2015; Yang et al., 2017), may, at least at diagnosis, not be integral to high-risk neuroblastoma. Interestingly, the role(s) of Hippo signaling in $\mathrm{NC}$ development are not well established, but the observations made here and by others are consistent with a report showing the importance of Hippo-YAP/TAZ in NC migration (Hindley et al., 2016).
The transcription factor PRRX1 was pinpointed as being involved in MES/NCC-like identity by both recent landmark studies on NB heterogeneity (Boeva et al., 2017; van Groningen et al., 2017). Indeed, the first of these directly demonstrated conversion of ADRN to MES lineages by PRRX1 induction in SK-N-BE(2)-C cells. As we had reported Wnt3a/Rspo2 treatment leading to EMT (Szemes et al., 2018), we investigated the possible links between Wnt signaling and PRRX1. As shown in Figure 4A, $P R R X 1$ transcripts were significantly higher in
A

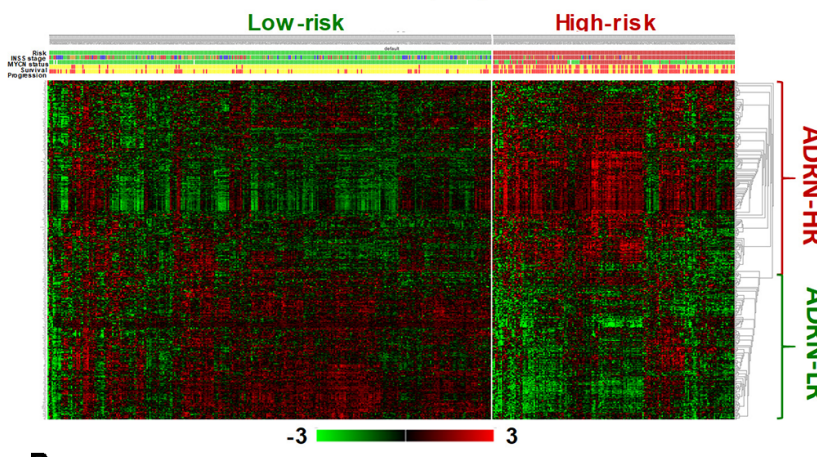

B

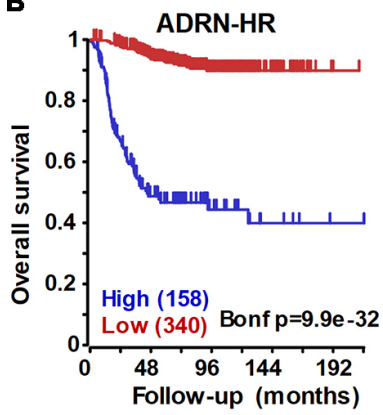

C
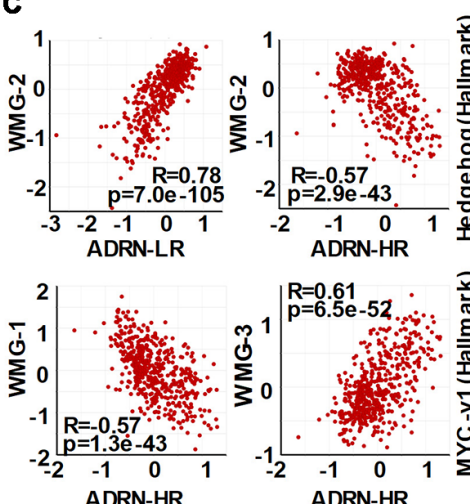

ADRN-LR

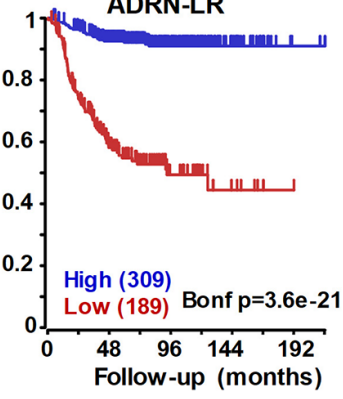

$\mathbf{E}$

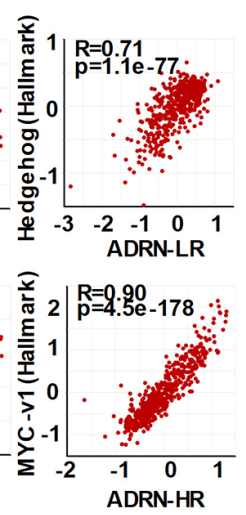

\section{Dersteeg/Etchevers mixed NB (34)}
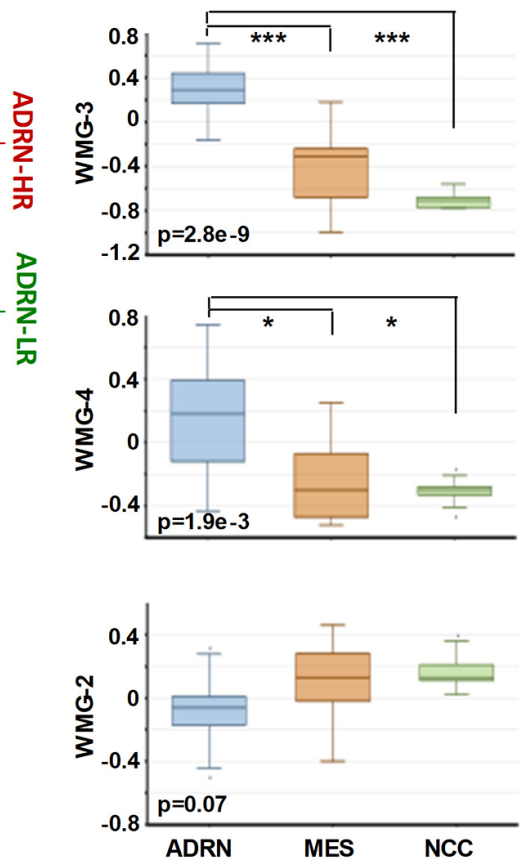

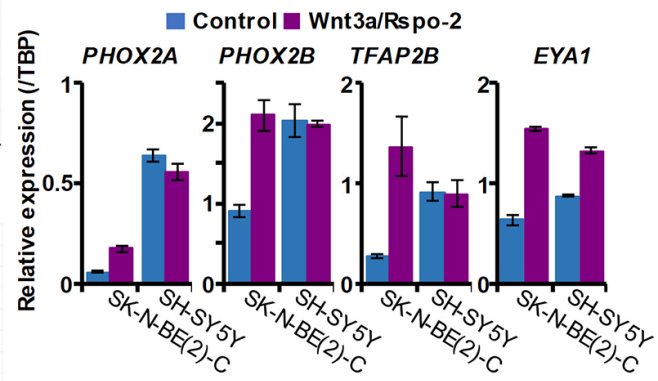

FIGURE 5 | The ADRN signature can be partitioned and the subgroups correlate with prognosis and neuroblastoma Wnt-regulated metagenes. (A) Heatmap showing the expression of ADRN signature genes in risk groups of SEQC NB set (GSE62564) reveals two subgroups. (B) Kaplan-Meier analysis demonstrating that the expression of the newly defined adrenergic low-risk (ADRN)-LR and adrenergic high-risk (HR) subgroups strongly and inversely correlate with prognosis. (C) WMG-2 shows a strong positive correlation with ADRN-LR, and negatively correlates with ADRN-HR. ADRN-LR has a positive association with Hedgehog signaling (Hallmark). ADRN-HR has a strong inverse correlation to WMG-1, and a positive correlation with WMG-3 and the expression of MYC targets-v1 (Hallmark). (D) WMG-3 and 4 are highly expressed in ADRN cells relative to MES and NC cells, while the expression of WMG-2 is not significantly different among the cell types (GSE14340 and GSE28019, van Groningen et al., 2017). Statistically significant difference between the groups was tested by using ANOVA ( $p$ values shown) and Tukey HSD post hoc tests were applied to evaluate pairwise differences (*** $\left.p<0.005,{ }^{*} p<0.05\right)$. (E) Long-term (96 h) Wnt3a/Rspo-2 treatment of SK-N-BE(2)-C cell can activate ADRN lineage transcription factors in NB cell lines. 
NB cluster 2 (intermediate risk, see Figure 2). Analyzing gene expression changes accompanying PRRX1 induction in SK-N$\mathrm{BE}(2)-\mathrm{C}$ cells, we found that WMG-1 genes, high expression of which is characteristic of intermediate risk NB (Szemes et al., 2018), showed increased expression following $144 \mathrm{~h}$ of $P R R X 1$ induction, with the exceptions of MSX2 and BMP4, which were increased at earlier timepoints (24-72 h). Expression of WMG-2 was also increased, although in a less uniform way, whereas WMG-3 and WMG-4 genes appeared to be partitioned in their response, with two subsets of genes regulated in an opposite fashion (Figure 4B). In sum, we found a remarkable overlap between our Wnt targets and the long-term transcriptional effects of PRRX1 induction in SK-N-BE(2)-C. We conducted the same analysis on Wnt pathway genes (KEGG and other known members) and observed increased expression of Wnt pathway receptor and transcriptional regulator genes accompanying PRRX1 induction (Supplementary Figure S2).

Although the kinetics of WMG responses argues against our Wnt genes being direct targets of PRRX1, this analysis links PRRX1, Wnt signaling and MES identity. We note that PRRX1 has been reported to be upstream of Wnt signaling and regulating EMT in gastric cancer (Guo et al., 2015). We also investigated whether Wnt signaling might form part of the regulatory circuit involving PRRX1, and the Hippo regulator TAZ (encoded by WWTR1), which were both implicated in MES/NCC-like identity. As these were not identified in our RNA sequencing timepoint $(6 \mathrm{~h})$, we assessed $96 \mathrm{~h}$ treatments of SK-N-BE(2)-C and SH-SY5Y cell lines by qPCR. Both PRRX1 and WWTR1 were upregulated by greater than 2-fold in both cell lines (Figure 4C), suggesting indirect regulation by Wnt signaling. Finally, as we found strong interrelationships between WMG-1 with Hippo-YAP/TAZ, and PRRX1, we tested the possible link between PRRX1 and Hippo signaling. Evaluation of the PRRX1 induction gene expression dataset revealed that numerous genes encoding for members of the Hippo signaling pathway were markedly upregulated, especially the Hippo effectors YAP1,WWTR1, their interacting transcription factors, SNAI2 and TEAD2, and the archetypal YAP/TAZ target gene CTGF (Figure 4D). Taken together, this analysis supports Wnt signaling as a major regulator of the MES/NCC-like identity evident in NB.

Wnt signaling is known to crosstalk with Hippo-YAP/TAZ signaling, and an alternative $\mathrm{Wnt}$ pathway has been characterized showing that a non-canonical Wnt-YAP/TAZ signaling can be initiated by Wnt3a or Wnt5a/b, which can then suppress the canonical Wnt/ $\beta$-catenin pathway (Park et al., 2015). Although Wnt3a can activate both canonical and non-canonical Wnt pathways (Samarzija et al., 2009), the majority of the genes we identified in SK-N-BE(2)-C cells had been shown to have $\beta$-catenin binding to their promoters (Szemes et al., 2018). Furthermore, we did not see early effects on YAP and TAZ proteins, or on CTGF expression (unpublished data) of Wnt3a/Rspo2 treatment in NB cells. Whilst this supports the early transcriptional response of SK-N-BE(2)-C cells to $\mathrm{Wnt} 3 \mathrm{a} / \mathrm{Rspo} 2$ to be canonical Wnt/ $\beta$-catenin signaling, we also note that the non-canonical Wnt ligand genes, WNT5B and WNT11, were amongst genes upregulated by our treatments

(Table 1). Thus, although it currently remains unclear what the relative contributions of canonical and non-canonical Wnt signaling are to NB cellular heterogeneity, it is evident that canonical Wnt signaling is interacting with other trans regulators to determine and maintain MES identity.

Our Wnt-driven NB phenotypes and transcriptome also indicated neural differentiation in SK-N-BE(2)-C and SH-SY5Y cell lines, including induction of NEFL and NEFM. In terms of NB cellular identities, this suggests that Wnt may also influence ADRN (or noradrenergic) cell fates. As a first step to analyzing this possibility, we determined the expression pattern of the ADRN signature genes in the four prognostic NB clusters defined by the expression of our NB Wnt target genes (Szemes et al., 2018; Supplementary Figure S3). ADRN genes had low expression in NB cluster 2, dominated by the overexpression of MES-related WMG-1. We also observed that the ADRN genes clustered into two subsets according to expression in the different prognostic Wnt groups. Therefore, we repeated the clustering in the SEQC dataset according to clinical risk categories and defined high-risk (ADRN-HR) and low-risk ADRN (ADRN-LR) signatures, based on high expression of the subsets in risk groups (Figure 5A). Contrasting expression of these two ADRN subsets was particularly striking in MNA tumors, which showed the highest expression of ADRN-HR and the lowest of ADRN-LR genes. Kaplan-Meier survival analyses showed that the ADRN-HR and ADRN-LR signatures were, respectively, strong negative and positive prognostic indicators (Figure 5B). We also found that ADRN-LR was positively correlated with WMG-2, which in turn negatively correlated with ADRN-HR. Evaluation of Hallmark genesets highlighted a striking positive correlation of ADRN-LR with

TABLE 2 | Gene ontology analysis of adrenergic low-risk (ADRN-LR) and adrenergic high-risk (HR) subgroups of genes.

\begin{tabular}{lllc}
\hline GO ID & Pathway description & N & FDR \\
\hline ADRN-LR & & & \\
G0.0007399 & Nervous system development & 52 & $6.16 \mathrm{E}-12$ \\
G0.0030182 & Neuron differentiation & 37 & $6.16 \mathrm{E}-12$ \\
G0.0048699 & Generation of neurons & 43 & $6.16 \mathrm{E}-12$ \\
G0.0022008 & Neurogenesis & 42 & $7.11 \mathrm{E}-11$ \\
G0.0048666 & Neuron development & 32 & $8.62 \mathrm{E}-11$ \\
G0.0031175 & Neuron projection development & 29 & $1.45 \mathrm{E}-10$ \\
G0.0048667 & Cell morphogenesis involved in neuron & 24 & $1.30 \mathrm{E}-08$ \\
& differentiation & & \\
G0.0048731 & System development & 64 & $1.30 \mathrm{E}-08$ \\
G0.0048812 & Neuron projection morphogenesis & 24 & $1.69 \mathrm{E}-08$ \\
G0.0000904 & Cell morphogenesis involved in & 26 & $2.12 \mathrm{E}-08$
\end{tabular}

\section{ADRN-HR}

GO.0000278

GO.1903047

G0.0022402

G0.0007067

G0.0000280

G0.0007049

G0.0044772

G0.0006268

G0.0006271

G0.0051301
Cell morphogenesis involved in differentiation

Mitotic cell cycle

Mitotic cell cycle process

Cell cycle process

Mitotic nuclear division

Nuclear division

Cell cycle

Mitotic cell cycle phase transition

DNA unwinding involved in DNA

replication

DNA strand elongation involved in DNA replication

Cell division
2.41E-06

2.72E-06

6.23E-06

1.77E-05

5.86E-05

5.86E-05

0.00085

0.00161

0.0104

0.012 
Hedgehog signaling, as we had previously pointed out for WMG-2 (Szemes et al., 2018; Figure 5C, top). The ADRN-HR signature negatively correlated with WMG-1, but positively with WMG-3, and accordingly, showed a very strong correlation with an MYC signature (Figure 5C, bottom). Gene Ontology analysis for ADRN-LR genes revealed enrichment for neuronal development and differentiation pathways, and in contrast, ADRN-HR genes were largely involved in cell-cycle processes, proliferation (Table 2). In keeping with the strong association between ADRN-HR and WMG-3 signatures, WMG-3 genes were expressed at significantly higher levels in ADRN cells compared to MES and NCC lines. WMG-4 displayed a similar expression pattern, albeit with lower significance (Figure 5D). WMG-2 was not significantly different between MES and ADRN cell lines but was slightly lower in $\mathrm{ADRN}$ lines relative to NCC lines.

Since we previously demonstrated that Wnt3a/Rspo2 treatment can prompt neural differentiation of NB cell lines, we evaluated whether long-term $(96 \mathrm{~h})$ treatments with Wnt ligands may influence transcription factors that contribute to the establishment of the ADRN, or noradrenergic, cell lineage. As shown in Figure 5E, $P H O X 2 A / 2 B, T F A P 2 B$ and $E Y A 1$ were all markedly upregulated in SK-N-BE(2)-C cells. In SH-SY5Y cells, these factors were already generally higher, but again induction of EYA1 was apparent following Wnt3a/Rspo 2 treatment.

Taken together these analyses demonstrate that the ADRN signature can be partitioned into two discrete subsets, which we have termed ADRN-LR and ADRN-HR, with contrasting expression in low-risk and high-risk NBs. The role of Wnt in differentiation of sympathoadrenergic neurons is further underlined by a report by Bodmer et al. (2009) that Wnt5a promotes axonal growth and branching in response to NGF:TrkA signaling. Our data also suggest co-operativity of Wnt with Hedgehog signaling in establishing the ADRN-LR signature. The role of Hedgehog signaling in NB remains poorly understood and, as in the case of Wnt signaling, there is conflicting evidence; two studies have suggested its oncogenic potential (Mao et al., 2009; Xu et al., 2012), whereas others have reported an influence on differentiation of NB cells (Williams et al., 2000; Souzaki et al., 2010). A role for Hedgehog signaling in cephalic and trunk NCC differentiation has been reported (Calloni et al., 2007), and in Xenopus, the Hedgehog transcription factor Gli2 was shown to be necessary for NCC specification and migration (Cerrizuela et al., 2018). In the context of $\mathrm{NB}$, our previous studies suggested that WMG-2 genes were correlated with Hedgehog signaling and are suppressed by MYCN (Szemes et al., 2018). It, therefore, follows that ADRN-LR genes, strongly associated with neural development, may be Wnt/Hedgehog-regulated genes repressed by MYCN. This informs and extends the suggestion that MYCN represses signaling pathways driving differentiation during NB tumorigenesis (Duffy et al., 2015).

\section{SUMMARY AND PERSPECTIVES}

The understanding of gene expression programmes, contingent on transcription factor hierarchies and chromatin level plasticity, in particular, epigenetic changes at super-enhancer elements, continues to facilitate our understanding of cellular identity. Notable advances have been made in ascertaining regulatory circuits in NCCs as well as in neuroblastoma recently, yet much remains to be determined about the contributions of autocrine, paracrine and juxtacrine signaling pathways that, together with transcription factors and epigenetic states, form a regulatory triumvirate.

Our analysis of Wnt signaling strongly suggests that this pathway is a major determinant of transcription factor circuitries, and thereby lineage identity, in neuroblastoma. This is further supported by comparisons with NC GRNs, with Wnt signaling regulating early NC specifiers such as $M S X 1$ and $D L X 5 / 6$, as well as migratory factors such as SNAI2 and TWIST1/2. Further, we highlight the possible interplay between canonical Wnt signaling with Hippo-YAP/TAZ and Hedgehog signaling, emphasizing the need for integrative analysis of signaling inputs for both $\mathrm{NC}$ and NB biology. Our transcriptomic signatures facilitate this, but also underline the need for parallel studies using diverse cell types and signaling effectors.

With regard to $\mathrm{NB}$, our study begins to reconcile some of the apparent contradictions about the influence of Wnt signaling. The determination of MES/NCC-like identity by Wnt is consistent with reports that resistance to chemotherapy and stemness may be linked to Wnt (Flahaut et al., 2009; Cantilena et al., 2011; Vangipuram et al., 2012). However, as we have shown, there are also Wnt-driven gene modules linked to neuronal differentiation. We propose that establishing a "Signaling Regulatory Network" will aid the formulation of a novel and efficacious treatment combinations for resistant neuroblastoma and differentiation therapies.

\section{AUTHOR CONTRIBUTIONS}

MS and KM designed and performed the analyses and experiments. All authors contributed to writing and producing the manuscript (MS, AG and KM).

\section{FUNDING}

We wish to thank Children with Cancer UK, the Childrens Cancer and Leukaemia Group (CCLG), Neuroblastoma UK and Smile with Siddy, the Biotechnology and Biological Sciences Research Council (BB/P008232/1) and Cancer Research UK (A12743/A21046; to KM) for funding this study.

\section{SUPPLEMENTARY MATERIAL}

The Supplementary Material for this article can be found online at: https://www.frontiersin.org/articles/10.3389/fnmol. 2019.00090/full\#supplementary-material

FIGURE S1 | (A) Correlation between MES signature and WMG-1 and (B) Kaplan-Meier survival analysis of MES genes in Kocak(649) data set (GSE45547).

TABLE S1 | Oligonucleotide primer sequences for qPCR. 


\section{REFERENCES}

Armstrong, A., Ryu, Y. K., Chieco, D., and Kuruvilla, R. (2011). Frizzled3 is required for neurogenesis and target innervation during sympathetic nervous system development. J. Neurosci. 31, 2371-2381. doi: 10.1523/jneurosci.424310.2011

Barembaum, M., and Bronner, M. E. (2013). Identification and dissection of a key enhancer mediating cranial neural crest specific expression of transcription factor, Ets-1. Dev. Biol. 382, 567-575. doi: 10.1016/j.ydbio.2013.08.009

Bodmer, D., Levine-Wilkinson, S., Richmond, A., Hirsh, S., and Kuruvilla, R. (2009). Wnt5a mediates nerve growth factor-dependent axonal branching and growth in developing sympathetic neurons. J. Neurosci. 29, 7569-7581. doi: 10.1523/jneurosci.1445-09.2009

Boeva, V., Louis-Brennetot, C., Peltier, A., Durand, S., Pierre-Eugene, C., Raynal, V., et al. (2017). Heterogeneity of neuroblastoma cell identity defined by transcriptional circuitries. Nat. Genet. 49, 1408-1413. doi: 10.1038/ ng.3921

Brodeur, G. M. (2003). Neuroblastoma: biological insights into a clinical enigma. Nat. Rev. Cancer 3, 203-216. doi: 10.1038/nrc1014

Brodeur, G. M., Seeger, R. C., Schwab, M., Varmus, H. E., and Bishop, J. M. (1984). Amplification of N-myc in untreated human neuroblastomas correlates with advanced disease stage. Science 224, 1121-1124. doi: 10.1126/science.67 19137

Bronner, M. E., and LeDouarin, N. M. (2012). Development and evolution of the neural crest: an overview. Dev. Biol. 366, 2-9. doi: 10.1016/j.ydbio.2011.12.042

Burstyn-Cohen, T., Stanleigh, J., Sela-Donenfeld, D., and Kalcheim, C. (2004). Canonical Wnt activity regulates trunk neural crest delamination linking BMP/noggin signaling with G1/S transition. Development 131, 5327-5339. doi: 10.1242/dev.01424

Calloni, G. W., Glavieux-Pardanaud, C., Le Douarin, N. M., and Dupin, E. (2007). Sonic Hedgehog promotes the development of multipotent neural crest progenitors endowed with both mesenchymal and neural potentials. Proc. Natl. Acad. Sci. U S A 104, 19879-19884. doi: 10.1073/pnas.0708806104

Cantilena, S., Pastorino, F., Pezzolo, A., Chayka, O., Pistoia, V., Ponzoni, M., et al. (2011). Frizzled receptor 6 marks rare, highly tumourigenic stem-like cells in mouse and human neuroblastomas. Oncotarget 2, 976-983. doi: 10.18632/oncotarget.410

Cerrizuela, S., Vega-Lopez, G. A., Palacio, M. B., Tribulo, C., and Aybar, M. J. (2018). Gli2 is required for the induction and migration of Xenopus laevis neural crest. Mech. Dev. 154, 219-239. doi: 10.1016/j.mod.2018.07.010

Ciccarone, V., Spengler, B. A., Meyers, M. B., Biedler, J. L., and Ross, R. A. (1989). Phenotypic diversification in human neuroblastoma cells: expression of distinct neural crest lineages. Cancer Res. 49, 219-225.

Clevers, H., and Nusse, R. (2012). Wnt/ $\beta$-catenin signaling and disease. Cell 149, 1192-1205. doi: 10.1016/j.cell.2012.05.012

de Lau, W., Barker, N., Low, T. Y., Koo, B. K., Li, V. S., Teunissen, H., et al. (2011). Lgr5 homologues associate with Wnt receptors and mediate R-spondin signalling. Nature 476, 293-297. doi: 10.1038/nature10337

Duffy, D. J., Krstic, A., Halasz, M., Schwarzl, T., Fey, D., Iljin, K., et al. (2015). Integrative omics reveals $\mathrm{MYCN}$ as a global suppressor of cellular signalling and enables network-based therapeutic target discovery in neuroblastoma. Oncotarget 6, 43182-43201. doi: 10.18632/oncotarget.6568

Duffy, D. J., Krstic, A., Schwarzl, T., Halasz, M., Iljin, K., Fey, D., et al. (2016). Wnt signalling is a bi-directional vulnerability of cancer cells. Oncotarget 7, 60310-60331. doi: 10.18632/oncotarget.11203

Espinosa-Medina, I., Saha, O., Boismoreau, F., Chettouh, Z., Rossi, F., Richardson, W. D., et al. (2016). The sacral autonomic outflow is sympathetic. Science 354, 893-897. doi: 10.1126/science.aah5454

Flahaut, M., Meier, R., Coulon, A., Nardou, K. A., Niggli, F. K., Martinet, D., et al. (2009). The Wnt receptor FZD1 mediates chemoresistance in neuroblastoma through activation of the $\mathrm{Wnt} / \beta$-catenin pathway. Oncogene 28, 2245-2256. doi: 10.1038 /onc. 2009.80

Fodde, R., and Tomlinson, I. (2010). Nuclear $\beta$-catenin expression and Wnt signalling: in defence of the dogma. J. Pathol. 221, 239-241. doi: 10.1002/ path. 2718

García-Castro, M. I., Marcelle, C., and Bronner-Fraser, M. (2002). Ectodermal Wnt function as a neural crest inducer. Science 297, 848-851. doi: $10.1126 /$ science. 1070824
Gherardi, S., Valli, E., Erriquez, D., and Perini, G. (2013). MYCN-mediated transcriptional repression in neuroblastoma: the other side of the coin. Front. Oncol. 3:42. doi: 10.3389/fonc.2013.00042

Grainger, S., and Willert, K. (2018). Mechanisms of Wnt signaling and control. Wiley Interdiscip. Rev. Syst. Biol. Med. doi: 10.1002/wsbm.1422 [Epub ahead of print].

Guo, J., Fu, Z., Wei, J., Lu, W., Feng, J., and Zhang, S. (2015). PRRX1 promotes epithelial-mesenchymal transition through the Wnt/ $\beta$-catenin pathway in gastric cancer. Med. Oncol. 32:393. doi: 10.1007/s12032-014-0393-x

Hao, H. X., Xie, Y., Zhang, Y., Charlat, O., Oster, E., Avello, M., et al. (2012). ZNRF3 promotes Wnt receptor turnover in an R-spondin-sensitive manner. Nature 485, 195-200. doi: 10.1038/nature11019

Hari, L., Brault, V., Kléber, M., Lee, H. Y., Ille, F., Leimeroth, R., et al. (2002). Lineage-specific requirements of $\beta$-catenin in neural crest development. J. Cell Biol. 159, 867-880. doi: 10.1083/jcb.200209039

Hindley, C. J., Condurat, A. L., Menon, V., Thomas, R., Azmitia, L. M., Davis, J. A., et al. (2016). The Hippo pathway member YAP enhances human neural crest cell fate and migration. Sci. Rep. 6:23208. doi: 10.1038/srep23208

Kerosuo, L., Neppala, P., Hsin, J., Mohlin, S., Vieceli, F. M., Torok, Z., et al. (2018) Enhanced expression of MycN/CIP2A drives neural crest toward a neural stem cell-like fate: implications for priming of neuroblastoma. Proc. Natl. Acad. Sci. U S A 115, E7351-E7360. doi: 10.1073/pnas.1800039115

Khudyakov, J., and Bronner-Fraser, M. (2009). Comprehensive spatiotemporal analysis of early chick neural crest network genes. Dev. Dyn. 238, 716-723. doi: 10.1002/dvdy.21881

Kocak, H., Ackermann, S., Hero, B., Kahlert, Y., Oberthuer, A., Juraeva, D., et al. (2013). Hox-C9 activates the intrinsic pathway of apoptosis and is associated with spontaneous regression in neuroblastoma. Cell Death Dis. 4:e586. doi: 10.1038/cddis.2013.84

Komiya, Y., and Habas, R. (2008). Wnt signal transduction pathways Organogenesis 4, 68-75. doi: 10.4161/org.4.2.5851

Leung, A. W., Murdoch, B., Salem, A. F., Prasad, M. S., Gomez, G. A., and GarciaCastro, M. I. (2016). WNT/ $\beta$-catenin signaling mediates human neural crest induction via a pre-neural border intermediate. Development 143, 398-410. doi: $10.1242 / \mathrm{dev} .130849$

Li, N., Fu, H., Hewitt, S. M., Dimitrov, D. S., and Ho, M. (2017). Therapeutically targeting glypican-2 via single-domain antibody-based chimeric antigen receptors and immunotoxins in neuroblastoma. Proc. Natl. Acad. Sci. U S A 114, E6623-E6631. doi: 10.1073/pnas.1706055114

Liu, X., Mazanek, P., Dam, V., Wang, Q., Zhao, H., Guo, R., et al. (2008). Deregulated Wnt/ $\beta$-catenin program in high-risk neuroblastomas without MYCN amplification. Oncogene 27, 1478-1488. doi: 10.1038/sj.onc.1210769

MacDonald, B. T., Tamai, K., and He, X. (2009). Wnt/ $\beta$-catenin signaling: components, mechanisms, and diseases. Dev. Cell 17, 9-26. doi: 10.1016/j. devcel.2009.06.016

Mao, L., Xia, Y. P., Zhou, Y. N., Dai, R. L., Yang, X., Duan, S. J., et al. (2009). A critical role of Sonic Hedgehog signaling in maintaining the tumorigenicity of neuroblastoma cells. Cancer Sci. 100, 1848-1855. doi: 10.1111/j.1349-7006. 2009.01262.x

Maris, J. M., Hogarty, M. D., Bagatell, R., and Cohn, S. L. (2007). Neuroblastoma Lancet 369, 2106-2120. doi: 10.1016/S0140-6736(07)60983-0

Martik, M. L., and Bronner, M. E. (2017). Regulatory logic underlying diversification of the neural crest. Trends Genet. 33, 715-727. doi: 10.1016/j. tig.2017.07.015

Matthay, K. K., Maris, J. M., Schleiermacher, G., Nakagawara, A., Mackall, C. L., Diller, L., et al. (2016). Neuroblastoma. Nat. Rev. Dis. Primers 2:16078. doi: $10.1038 /$ nrdp. 2016.78

Mayor, R., and Theveneau, E. (2014). The role of the non-canonical Wnt-planar cell polarity pathway in neural crest migration. Biochem. J. 457, 19-26. doi: 10.1042/bj20131182

Nieto, M. A., Huang, R. Y., Jackson, R. A., and Thiery, J. P. (2016). Emt: 2016. Cell 166, 21-45. doi: 10.1016/j.cell.2016.06.028

Nikitina, N., Sauka-Spengler, T., and Bronner-Fraser, M. (2008). Dissecting early regulatory relationships in the lamprey neural crest gene network. Proc. Natl. Acad. Sci. U S A 105, 20083-20088. doi: 10.1073/pnas.0806009105

Nusse, R., and Clevers, H. (2017). Wnt/ $\beta$-catenin signaling, disease, and emerging therapeutic modalities. Cell 169, 985-999. doi: 10.1016/j.cell.2017. 05.016 
Olsen, R. R., Otero, J. H., Garcia-Lopez, J., Wallace, K., Finkelstein, D., Rehg, J. E., et al. (2017). MYCN induces neuroblastoma in primary neural crest cells. Oncogene 36, 5075-5082. doi: 10.1038/onc.2017.128

Park, H. W., Kim, Y. C., Yu, B., Moroishi, T., Mo, J. S., Plouffe, S. W., et al. (2015). Alternative Wnt signaling activates YAP/TAZ. Cell 162, 780-794. doi: $10.1016 /$ j.cell.2015.07.013

Ramos, C., and Robert, B. (2005). msh/Msx gene family in neural development. Trends Genet. 21, 624-632. doi: 10.1016/j.tig.2005.09.001

Samarzija, I., Sini, P., Schlange, T., Macdonald, G., and Hynes, N. E. (2009). Wnt3a regulates proliferation and migration of HUVEC via canonical and non-canonical Wnt signaling pathways. Biochem. Biophys. Res. Commun. 386, 449-454. doi: 10.1016/j.bbrc.2009.06.033

Sharpe, P. T. (2016). Dental mesenchymal stem cells. Development 143, 2273-2280. doi: 10.1242/dev.134189

Souzaki, R., Tajiri, T., Souzaki, M., Kinoshita, Y., Tanaka, S., Kohashi, K., et al. (2010). Hedgehog signaling pathway in neuroblastoma differentiation. J. Pediatr. Surg. 45, 2299-2304. doi: 10.1016/j.jpedsurg.2010.08.020

Steventon, B., Araya, C., Linker, C., Kuriyama, S., and Mayor, R. (2009). Differential requirements of BMP and Wnt signalling during gastrulation and neurulation define two steps in neural crest induction. Development 136, 771-779. doi: 10.1242/dev.029017

Stuhlmiller, T. J., and García-Castro, M. I. (2012). FGF/MAPK signaling is required in the gastrula epiblast for avian neural crest induction. Development 139, 289-300. doi: 10.1242/dev.070276

Su, Z., Fang, H., Hong, H., Shi, L., Zhang, W., Zhang, W., et al. (2014). An investigation of biomarkers derived from legacy microarray data for their utility in the RNA-seq era. Genome Biol. 15:523. doi: 10.1186/s13059-014-0523-y

Szemes, M., Greenhough, A., Melegh, Z., Malik, S., Yuksel, A., Catchpoole, D., et al. (2018). Wnt signalling drives context-dependent differentiation or proliferation in neuroblastoma. Neoplasia 20, 335-350. doi: 10.1016/j.neo.2018. 01.009

ten Berge, D., Brugmann, S. A., Helms, J. A., and Nusse, R. (2008). Wnt and FGF signals interact to coordinate growth with cell fate specification during limb development. Development 135, 3247-3257. doi: 10.1242/dev.023176

Tribulo, C., Aybar, M. J., Nguyen, V. H., Mullins, M. C., and Mayor, R. (2003). Regulation of Msx genes by a Bmp gradient is essential for neural crest specification. Development 130, 6441-6452. doi: 10.1242/dev.00878

van Amerongen, R. (2012). Alternative Wnt pathways and receptors. Cold Spring Harb. Perspect. Biol. 4:a007914. doi: 10.1101/cshperspect.a007914

Vangipuram, S. D., Buck, S. A., and Lyman, W. D. (2012). Wnt pathway activity confers chemoresistance to cancer stem-like cells in a neuroblastoma cell line. Tumour Biol. 33, 2173-2183. doi: 10.1007/s13277-012-0478-0

van Groningen, T., Koster, J., Valentijn, L. J., Zwijnenburg, D. A., Akogul, N., Hasselt, N. E., et al. (2017). Neuroblastoma is composed of two super-enhancerassociated differentiation states. Nat. Genet. 49, 1261-1266. doi: 10.1038/ ng.3899
Veeman, M. T., Axelrod, J. D., and Moon, R. T. (2003). A second canon. Functions and mechanisms of $\beta$-catenin-independent Wnt signaling. Dev. Cell 5, 367-377. doi: 10.1016/S1534-5807(03)00266-1

Vieira, G. C., Chockalingam, S., Melegh, Z., Greenhough, A., Malik, S., Szemes, M., et al. (2015). LGR5 regulates pro-survival MEK/ERK and proliferative Wnt/ $\beta$-catenin signalling in neuroblastoma. Oncotarget 6, 40053-40067. doi: 10.18632/oncotarget.5548

Wakamatsu, Y., Watanabe, Y., Nakamura, H., and Kondoh, H. (1997). Regulation of the neural crest cell fate by N-myc: promotion of ventral migration and neuronal differentiation. Development 124, 1953-1962.

Wang, M., Liu, Y., Zou, J., Yang, R., Xuan, F., Wang, Y., et al. (2015). Transcriptional co-activator TAZ sustains proliferation and tumorigenicity of neuroblastoma by targeting CTGF and PDGF- $\beta$. Oncotarget 6, 9517-9530. doi: 10.18632/oncotarget.3367

Westermark, U. K., Wilhelm, M., Frenzel, A., and Henriksson, M. A. (2011). The MYCN oncogene and differentiation in neuroblastoma. Semin. Cancer Biol. 21, 256-266. doi: 10.1016/j.semcancer.2011.08.001

Williams, Z., Tse, V., Hou, L., Xu, L., and Silverberg, G. D. (2000). Sonic hedgehog promotes proliferation and tyrosine hydroxylase induction of postnatal sympathetic cells in vitro. Neuroreport 11,3315-3319. doi: 10.1097/00001756200010200-00011

Xu, P., Dang, Y., Wang, L., Liu, X., Ren, X., Gu, J., et al. (2016). Lgr4 is crucial for skin carcinogenesis by regulating MEK/ERK and $\mathrm{Wnt} / \beta$-catenin signaling pathways. Cancer Lett. 383, 161-170. doi: 10.1016/j.canlet.2016.09.005

Xu, L., Wang, X., Wan, J., Li, T., Gong, X., Zhang, K., et al. (2012). Sonic Hedgehog pathway is essential for neuroblastoma cell proliferation and tumor growth. Mol. Cell. Biochem. 364, 235-241. doi: 10.1007/s11010-011-1222-6

Yang, C., Tan, J., Zhu, J., Wang, S., and Wei, G. (2017). YAP promotes tumorigenesis and cisplatin resistance in neuroblastoma. Oncotarget 8, 37154-37163. doi: 10.18632/oncotarget.16209

Zins, K., Schäfer, R., Paulus, P., Dobler, S., Fakhari, N., Sioud, M., et al. (2016). Frizzled 2 signaling regulates growth of high-risk neuroblastomas by interfering with $\beta$-catenin-dependent and $\beta$-catenin-independent signaling pathways. Oncotarget 7, 46187-46202. doi: 10.18632/oncotarget.10070

Conflict of Interest Statement: The authors declare that the research was conducted in the absence of any commercial or financial relationships that could be construed as a potential conflict of interest.

Copyright (C) 2019 Szemes, Greenhough and Malik. This is an open-access article distributed under the terms of the Creative Commons Attribution License (CC BY). The use, distribution or reproduction in other forums is permitted, provided the original author(s) and the copyright owner(s) are credited and that the original publication in this journal is cited, in accordance with accepted academic practice. No use, distribution or reproduction is permitted which does not comply with these terms. 